\title{
Low and Medium dose Statins Improve Function and Prognosis of Asian Ischemic Stroke Patients with Intravenous Thrombolysis
}

\section{Chaohua Cui}

Department of Neurology, West China Hospital, Sichuan University, Chengdu, China Jiajia Bao

Department of Neurology, West China Hospital, Sichuan University, Chengdu, China Lijie Gao

Department of Neurology, West China Hospital, Sichuan University, Chengdu, China Li He ( $\nabla$ heli2003new@126.com )

Department of Neurology, West China Hospital, Sichuan University, Chengdu, China

\section{Research Article}

Keywords: stroke, statins, intravenous thrombolysis, efficacy and safety outcome

Posted Date: February 16th, 2021

DOI: https://doi.org/10.21203/rs.3.rs-198975/v1

License: (a) (i) This work is licensed under a Creative Commons Attribution 4.0 International License. Read Full License 


\section{Abstract}

Background: For acute ischaemic stroke, intravenous thrombolysis combined with statins might increase the therapeutic effect; however, it is uncertain whether this is effective. Additionally, statins can increase the risk of intracerebral haemorrhage in ischaemic stroke patients, further raising doubts regarding the safety of this combination.

Methods: Data from consecutive acute ischaemic stroke patients with intravenous thrombolysis were prospectively collected. Efficacy outcomes included NIHSS (National Institutes of Health Stroke Scale) score improvement at 7 days after admission and mRS (Modified Rankin Scale) improvement at 90 days. Safety outcomes included haemorrhage events in the hospital and death events within 2 years.

Results: The study finally included 222 patients. The statin group had a higher percentage of NIHSS improvement at 7 days $(p<0.001)$ and a higher percentage of a favourable functional outcome (FFO) $(p<0.001)$ at 90 days. The statin group had a lower percentage of intracerebral haemorrhage $(p<0.001)$ and gastrointestinal haemorrhage $(p=0.004)$ in the hospital and a lower percentage of death events $(p<0.001)$ within 2 years. Logistic regression indicated that statin use was significantly related to NIHSS improvement $(\mathrm{OR}=2.291, \mathrm{p}=0.014)$, a lower percentage of intracerebral haemorrhage $(\mathrm{OR}=0.379, \mathrm{p}=0.008)$ and gastrointestinal haemorrhage $(\mathrm{OR}=0.027, \mathrm{p}=0.023)$, and a lower percentage of death events $(\mathrm{OR}=0.196, \mathrm{p}<0.001)$.

Conclusion: For Asian acute ischaemic stroke patients after intravenous thrombolysis, the use of low- and medium-dose statins was related to NIHSS improvement of moderate stroke patients at 7 days, with a reduced percentage of haemorrhage events in the hospital and a lower percentage of death events within 2 years, especially for moderate stroke or noncardioembolic stroke patients.

\section{Background}

For acute ischaemic stroke within the time windows, IV thrombolysis (intravenous thrombolysis) is a clinically effective and guideline-recommended therapeutic method ${ }^{1}$. After intravenous thrombolysis, statins are regularly used as medicine for ischaemic stroke patients during hospitalization ${ }^{2}$. Intravenous thrombolysis therapy promotes recanalization of occluded vessels by dissolving thrombi in the acute phase $^{3}$. Statins inhibit thrombogenesis and the development of collaterals for ischaemic stroke ${ }^{4}$. Therefore, intravenous thrombolysis combined with statins might increase the therapeutic effect for ischaemic stroke patients.

However, two studies showed different conclusions regarding whether intravenous thrombolysis combined with statins was related to functional outcomes in ischaemic stroke patients. Manuel Cappellari's study suggested that statin use in the acute phase of stroke after IV thrombolysis may improve functional outcomes ${ }^{5}$. In contrast, Ashkan Mowla's study suggested that thrombolysis combined with statins had no significant association with the outcome of ischaemic stroke ${ }^{6}$. Therefore, it is 
uncertain whether intravenous thrombolysis combined with statins is effective for ischaemic stroke patients.

The fact that high-dose statins could increase the risk of intracerebral haemorrhage in ischaemic stroke patients further raises doubts about the safety of intravenous thrombolysis combined with statins ${ }^{4}$. Although the studies by Manuel Cappellari and Ashkan Mowla both showed that using statins did not increase the risk of intracerebral haemorrhage in ischaemic stroke patients after thrombolysis, these studies were conducted in Europe and America ${ }^{5,6}$. European and American stroke patients regularly take high-dose statins. However, Asian stroke patients often take low- and medium-dose statins. Therefore, the conclusions may not be suitable for Asian patients. The two studies were retrospective studies and did not have long-term follow-up data of more than 3 months.

The present prospective observational cohort study aimed to further explore the relationship between using medium- or low-dose statins combined with intravenous thrombolysis and the effects and safety outcomes of Asian ischaemic stroke patients.

\section{Methods}

\section{Participants}

We consecutively recruited patients with acute ischaemic stroke who received IV thrombolysis in the Neurology Department of West China Hospital, Sichuan University. The patients were enrolled from November 1, 2018, to July 31, 2020 and followed up from February 1, 2019, to October 31, 2020. The study is a prospective observational cohort design. The patients receiving statins in the hospital were assigned to the low- or medium-dose statin group by the dose of statins. Low-dose statins were defined as atorvastatin 10-20 mg per day, simvastatin $10 \mathrm{mg}$ per day, or rosuvastatin $5 \mathrm{mg}$ per day. Medium-dose statins were defined as atorvastatin $40 \mathrm{mg}$ per day, simvastatin $20 \mathrm{mg}$ per day, or rosuvastatin $10 \mathrm{mg}$ per day. The other patients were assigned to the control group. A total of 220 patients were needed to show a significant difference ${ }^{7}$.

The inclusion criteria were as follows: (1) age over 18 years; (2) acute ischaemic stroke patients with a diagnosis of stroke in accordance with WHO diagnostic criteria with evidence of neuroimaging; and (3) IV thrombolysis therapy administration within 4.5 hours after stroke onset.

The exclusion criteria were as follows: (1) mRS was $\geq 2$ before onset; (2) the time between admission and onset was more than 4.5 hours; (3) stroke associated with trauma or surgery; and (4) intracerebral haemorrhage, subarachnoid haemorrhage, coagulopathy, cancer, cardiac failure, or severe hepatic or renal dysfunction.

The study was performed in accordance with the Declaration of Helsinki and the ethical standards of the institutional and/or national research committee. The study was approved by the Ethics Committee of West China Hospital, Sichuan University with approval number 2019 (319). 


\section{Definition of risk factors}

Cardioembolic stroke was proven by definite history, electrocardiography, and cardiac colour ultrasound. ICH (intracerebral haemorrhage) was proven by CT in the hospital regardless of whether the condition of the patient changed. Gastrointestinal haemorrhage included haematemesis and haematochezia. An mRS score of 0-2 was defined as a favourable functional outcome (FFO) at 90 days. A difference in NIHSS score greater than 4 was defined as an improvement. A NIHSS score of 0-4 was defined as a mild stroke, a NIHSS score of 5-15 was defined as a moderate stroke, and a NIHSS score of $16-40$ was defined as a severe stroke.

\section{Data collection and outcome}

The baseline data were collected from electronic clinical records, and structured questionnaires were completed by patients or their relatives at the patients' admission to the hospital. The data included age, sex, blood pressure, history of smoking and drinking, NIHSS score and mRS score at admission, history of diseases and history of drug use. Types of strokes and types of statins were also collected. We recorded laboratory data, including TC (total cholesterol), TG (triglyceride), HDL-C (high-density lipoprotein cholesterol), and LDL-C. at the time of hospital admission.

The efficacy outcome was the difference in the NIHSS score between admission and 7 days after admission and FFO (mRS<=2) at 90 days after onset. The safety outcomes were intracerebral haemorrhage and gastrointestinal haemorrhage in the hospital and death events within 2 years after onset. We collected the NIHSS scores and in the hospital via face-to-face interviews. We collected the haemorrhage in the hospital via electronic clinical records. We collected the mRS scores at 90 days stroke onset and death events within 2 years via telephone (more than two different numbers of patients or relatives), WeChat (an instant messaging application) or e-mail. We collected death events at 3, 6, 12 and 24 months after discharge. The two experienced neurologists who evaluated events and outcomes were blind to the patients' condition and grouping.

\section{Statistical analysis}

To describe the baseline characteristics, the continuous variables were expressed as the mean and SD (standard deviation) or median and frequencies. Categorized data and ranked data are expressed as numbers and percentages. To compare the group differences, variance analysis was conducted for continuous data following the normal distribution. A nonparametric test (Mann-Whitney U test) was conducted for continuous data that did not follow a normal distribution. A chi-square test was conducted for categorical data and ranked data.

To analyse the outcome events, we compared the group differences using the chi-square test. We analysed the effects of risk factors on the outcome events using univariable and multivariable logistic 
regression methods. The eligible factors of multivariable logistic regression included (1) P of univariable logistic regression less than 0.1 and (2) a factor with clinical significance. We calculated the odds ratios (ORs), 95\% Cls and p values using logistic regression methods. We compared the death events at different times with a Kaplan-Meier curve. We performed subgroup analysis via univariable logistic regression by differences in NIHSS scores at admission and differences in stroke type (cardioembolic stroke and non-cardioembolic stroke). The threshold for statistical significance was set as $\mathrm{P}<0.05$. SPSS 23.0 for Windows was used to process the data.

\section{Results}

\section{Patients}

The study included 222 patients (120 in the low-dose statin group, 66 in the medium-dose statin group and 36 in the control group) whose baseline data were analysed. We lost 17 patients' data to follow-up, and the partial outcome data of 205 patients were acquired.

Among the 222 patients (mean age: $70.77 \pm 12.475$ years), 109 (49.1\%) were male. The median and interquartile ranges of the NIHSS at admission were 8 (5-14). Comparing the baseline data of the different groups, the low- and medium-dose statin groups had a higher percentage of antiplatelet drug use in the hospital, a lower percentage of cardioembolic and anticoagulation drug use in the hospital, and lower NIHSS scores at admission than the control group. No significant difference in other baseline data was found between the two groups (Table 01). 
Baseline characteristic

\begin{tabular}{|c|c|c|c|c|}
\hline variables & $\begin{array}{l}\text { Low dose group(n } \\
=120)\end{array}$ & $\begin{array}{l}\text { Medium dose } \\
\text { group }(n=66)\end{array}$ & $\begin{array}{l}\text { Control group } \\
(n=36)\end{array}$ & P* \\
\hline Age, years, mean(SD) & 70.93(13.007) & 69.86(10.318) & 71.92(14.377) & 0.225 \\
\hline Male,\% & $58(48.3)$ & $33(50)$ & $18(50)$ & 0.981 \\
\hline $\begin{array}{l}\text { Admission NIHSS score, } \\
\text { Med(IQR) }\end{array}$ & $8(5-15)$ & $8(4-13)$ & $15(8-18)$ & 0.005 \\
\hline $\begin{array}{l}\text { antiplatelet drug in } \\
\text { hospital,\% }\end{array}$ & $105(87.5)$ & $61(92.4)$ & $8(22.2)$ & <. \\
\hline cardioembolic,\% & $50(41.7)$ & 13(19.7) & $21(58.3)$ & $\begin{array}{l}< \\
0.001\end{array}$ \\
\hline $\begin{array}{l}\text { Anticoagulation in } \\
\text { hospital,\% }\end{array}$ & $38(31.7)$ & $8(12.1)$ & $18(50)$ & $<.001$ \\
\hline $\begin{array}{l}\text { systolic blood pressure } \\
\text { mmHg, mean(SD) }\end{array}$ & $147.95(25.045)$ & $149.42(23.813)$ & 153.81(31.962) & 0.494 \\
\hline $\begin{array}{l}\text { diastolic blood pressure } \\
\mathrm{mmHg} \text {, mean(SD) }\end{array}$ & $83.46(16.357)$ & $86.14(15.685)$ & $85.19(17.214)$ & 0.544 \\
\hline smoking, $\%$ & $37(30.8)$ & $27(40.9)$ & $10(27.8)$ & 0.291 \\
\hline hypertension,\% & $54(45)$ & $32(48.5)$ & $18(50)$ & 0.827 \\
\hline diabetes mellitus, $\%$ & $24(20)$ & $10(15.2)$ & $8(22.2)$ & 0.958 \\
\hline coronary heart disease,\% & $17(14.2)$ & $5(7.6)$ & $4(11.1)$ & 0.406 \\
\hline atrial fibrillation, $\%$ & $38(31.7)$ & $23(34.8)$ & $18(50)$ & 0.132 \\
\hline antiplatelet drug, $\%$ & $10(8.3)$ & $4(6.1)$ & $2(5.6)$ & 0.411 \\
\hline antihypertensive,\% & $33(27.5)$ & 13(19.7) & 12(33.3) & 0.425 \\
\hline hypoglycaemic,\% & 18(15) & $6(9)$ & $6(16.7)$ & 0.574 \\
\hline anticoagulation,\% & $8(6.7)$ & $2(3)$ & $3(8.3)$ & 0.757 \\
\hline Platelet, mmol/l, mean(SD) & 177.34(52.902) & $184.91(52.469)$ & $173.97(57.457)$ & 0.385 \\
\hline INR, mean(SD) & $0.98(0.090)$ & $0.97(0.127)$ & $0.99(0.111)$ & 0.411 \\
\hline
\end{tabular}

$P^{*}$ was calculated by ANOVA, Chi-square test, or Mann-Whitney $U$ test as appropriate. Mean value, SD standard distance, Med median value, IQR interquartile range, INR international normalized ratio, ALT glutamic-pyruvic transaminase, HDL-C high-density lipoprotein cholesterol, LDL-C low-density lipoprotein 


\begin{tabular}{|c|c|c|c|c|}
\hline variables & $\begin{array}{l}\text { Low dose group(n } \\
=120)\end{array}$ & $\begin{array}{l}\text { Medium dose } \\
\text { group }(n=66)\end{array}$ & $\begin{array}{l}\text { Control group } \\
(n=36)\end{array}$ & $\mathrm{P*}$ \\
\hline ALT, mmol/l, mean(SD) & 21.66(13.933) & $22.41(9.387)$ & $21.86(12.836)$ & 0.203 \\
\hline $\begin{array}{l}\text { Creatinine, mmol/l, } \\
\text { mean(SD) }\end{array}$ & $78.43(28.726)$ & $77.62(25.155)$ & $82.22(28.129)$ & 0.508 \\
\hline $\begin{array}{l}\text { Glucose, } \mathrm{mmol} / \mathrm{l}, \\
\text { mean(SD) }\end{array}$ & $7.83(2.511)$ & $7.74(2.732)$ & $7.99(3.397)$ & 0.791 \\
\hline $\begin{array}{l}\text { Triglyceride, } \mathrm{mmol} / \mathrm{l}, \\
\text { mean(SD) }\end{array}$ & $1.80(1.491)$ & $1.65(1.336)$ & $1.55(0.987)$ & 0.874 \\
\hline $\begin{array}{l}\text { Total cholesterol, } \mathrm{mmol} / \mathrm{l}, \\
\text { mean(SD) }\end{array}$ & $4.57(1.089)$ & $4.45(0.960)$ & $4.19(0.908)$ & 0.158 \\
\hline $\mathrm{HDL}-\mathrm{C}, \mathrm{mmol} / \mathrm{l}, \operatorname{mean}(\mathrm{SD})$ & $1.27(0.385)$ & $1.33(0.405)$ & $1.31(0.446)$ & 0.673 \\
\hline LDL-C, mmol/l, mean(SD) & $2.67(0.962)$ & $2.70(0.771)$ & $2.41(0.694)$ & 0.238 \\
\hline
\end{tabular}

The types of statins included simvastatin $(n=2)$, atorvastatin $(n=141)$, and rosuvastatin $(n=43)$, and the type of statins in the different dose statin groups was not significantly different (Table 02).

Table 02

Difference type of statins

\begin{tabular}{|llll|}
\hline Type of statins & Low dose group $(\mathbf{n}=120)$ & Medium dose group $(\mathbf{n = 6 6 )}$ & $\mathbf{P *}$ \\
\hline atorvastatin,\% & $95(79.2)$ & $48(72.7)$ & 0.141 \\
\hline rosuvastatin,\% & $25(20.8)$ & $16(24.2)$ & 0.141 \\
\hline simvastatin,\% & $0(0)$ & $2(3.1)$ & 0.141 \\
\hline$P^{*}$ was calculated by Chi-square test. & & \\
\hline
\end{tabular}

\section{Efficacy outcome}

We found that the low- $(p<0.001)$ and medium-dose $(p<0.001)$ statin groups had a higher percentage of improvement at 7 days than the control group, and the low- and medium-dose statin groups did not show a significant difference $(p=0.05)$. The low- $(p=0.027)$ and medium-dose $(p=0.003)$ statin groups had a higher percentage of FFOs at 90 days $(p=0.027)$ than the control group, and the low- and medium-dose statin groups did not show a significant difference $(p=0.246)$. (Table 03). 
Table 03

Outcome Comparation

\begin{tabular}{|c|c|c|c|c|c|c|c|}
\hline outcome & Control & $\begin{array}{l}\text { Low } \\
\text { dose }\end{array}$ & $\begin{array}{l}\text { Medium } \\
\text { dose }\end{array}$ & P1 & P2 & P3 & P4 \\
\hline NIHSS improvement,\% & $41.7 \%$ & $83.3 \%$ & $75.8 \%$ & 0.000 & 0.000 & 0.000 & 0.050 \\
\hline $\mathrm{FFO}, \%$ & $42.9 \%$ & $63.8 \%$ & $73.4 \%$ & 0.000 & 0.027 & 0.003 & 0.246 \\
\hline $\mathrm{ICH}, \%$ & $44.4 \%$ & $13.3 \%$ & $4.5 \%$ & 0.000 & 0.000 & 0.000 & 0.058 \\
\hline $\begin{array}{l}\text { gastrointestinal } \\
\text { haemorrhage,\% }\end{array}$ & $11.1 \%$ & $0.8 \%$ & $0 \%$ & 0.004 & 0.010 & 0.014 & 1.000 \\
\hline Death within 2 years,\% & $33.3 \%$ & $9.2 \%$ & $6.1 \%$ & 0.000 & 0.002 & 0.001 & 0.348 \\
\hline
\end{tabular}

In the univariate logistic regression analysis, we found that the use of statins $(\mathrm{OR}=3.640, \mathrm{P}<0.001)$ and the use of antiplatelet drugs in the hospital $(O R=2.892, \mathrm{P}<0.001)$ were related to a higher percentage of NIHSS score improvement at 7 days after admission (Fig. 01). Using statins (OR $=1.882, P=0.004)$ and the use of antiplatelet drugs in hospitals $(\mathrm{OR}=1.512, \mathrm{P}=0.008)$ were associated with FFO at 90 days. Higher NIHSS at admission ( $\mathrm{OR}=0.956, \mathrm{P}=0.037)$, higher NIHSS at 7 days after admission $(\mathrm{OR}=0.938$, $P<0.001)$, older age $(O R=0.975, P=0.039)$, higher systolic pressure $(O R=0.985, P=0.007)$ and $I C H$ in the hospital $(\mathrm{OR}=0.452, \mathrm{P}=0.036)$ were inversely related to FFO at 90 days (Fig. 01).

In the multivariable logistic regression analysis, after adjusting for risk factors, we found that the use of statins $(O R=2.291, P=0.014)$ and the use of antiplatelet drugs in the hospital $(O R=2.039, P=0.012)$ were still related to a higher percentage of NIHSS score improvement at 7 days after admission (Fig. 02). Higher NIHSS at 7 days after admission $(\mathrm{OR}=0.952, \mathrm{P}=0.009)$ and higher systolic pressure $(\mathrm{OR}=0.986$, $P=0.025$ ) were still significantly inversely related to FFO at 90 days (Fig. 02).

\section{Safety outcome}

When we evaluated $\mathrm{ICH}$ and gastrointestinal haemorrhage events in the hospital, we found that the low$(p<0.001)$ and medium-dose $(p<0.001)$ statin groups had a lower percentage of events than the control group, and the low- and medium-dose statin groups had no significant difference $(p=0.058)$. Regarding death events at the 2-year follow-up, we found that the low- $(p=0.002)$ and medium-dose $(p=0.001)$ statin groups had a lower percentage than the control group, and the low- and medium-dose statin groups had no significant difference $(p=0.348)$. (Table 03).

In the univariate logistic regression analysis, we found that the use of statins $(O R=0.224, P<0.001)$, use of antiplatelet drugs in the hospital $(\mathrm{OR}=0.270, \mathrm{P}<0.001)$ and higher value of platelets $(\mathrm{OR}=0.989, \mathrm{P}=$ 0.010) were inversely related to a higher percentage of $\mathrm{ICH}$ in the hospital (Fig. 03). Cardioembolic stroke 
$(O R=2.096, P=0.049)$ and a higher value of $L D L-C(O R=2.791, P=0.020)$ were related to a higher percentage of $\mathrm{ICH}$ in the hospital (Fig. 03). Using statins $(\mathrm{OR}=0.062, \mathrm{P}=0.011)$ was inversely related to a higher percentage of gastrointestinal haemorrhage in the hospital (Fig. 3). Older age (OR $=1.193, P=$ $0.019)$ and a higher value of blood glucose $(O R=1.220, P=0.019)$ were related to a higher percentage of gastrointestinal haemorrhage in the hospital (Fig. 03). We also found that the use of statins $(O R=0.110$, $P<0.001)$ and the use of antiplatelet drugs in hospitals $(O R=0.302, P<0.001)$ were inversely related to a higher percentage of death events within 2 years. Older age $(O R=1.061, P=0.002)$, higher NIHSS at admission $(\mathrm{OR}=1.073, \mathrm{P}=0.005)$ and higher $\mathrm{NIHSS}$ at 7 days after admission $(\mathrm{OR}=1.130, \mathrm{P}<0.001)$ were related to a higher percentage of death events within 2 years (Fig. 03).

In the multivariable logistic regression analysis, after adjusting for risk factors, we found that the use of statins $(O R=0.379, P=0.008)$, use of antiplatelet drugs in the hospital $(O R=0.395, P=0.003)$ and higher value of platelets $(\mathrm{OR}=0.990, \mathrm{P}=0.028)$ were still inversely related to a higher percentage of intracerebral haemorrhage in the hospital (Fig. 04). A higher value of $L D L-C(O R=3.133, P=0.033)$ was still related to a higher percentage of $\mathrm{ICH}$ in the hospital. Using statins $(\mathrm{OR}=0.027, \mathrm{P}=0.023)$ was still inversely related to a higher percentage of gastrointestinal haemorrhage in the hospital. Older age $(O R=1.413, P=0.027)$ and a higher value of blood glucose $(O R=1.399, P=0.029)$ were related to a higher percentage of gastrointestinal haemorrhage in the hospital (Fig. 04). Using statins $(O R=0.196, P<0.001)$ was still inversely associated with a higher percentage of death events within 2 years. Older age $(O R=1.054, P=$ $0.015)$ and higher NIHSS at 7 days after admission $(O R=1.093, P<0.001)$ were still related to a higher percentage of death events at 2 years (Fig. 04).

When we analysed death events in the different groups at different times with Kaplan-Meier curves, we found that the low- and medium-dose statin groups had a significantly higher survival rate than the control group at different times. The low-dose and medium-dose groups had similar survival rates within 20 months. After 20 months, the medium-dose group had a higher survival rate than the low-dose group (Fig. 05).

In the subgroup analysis, in mild stroke patients, statin use was only significantly inversely related to ICH $(\mathrm{OR}=0.018)$ in the hospital. Using statins were not related to other outcomes. In moderate stroke patients, using statins was significantly related to NIHSS improvement $(\mathrm{OR}=2.886)$ and was significantly inversely related to $\mathrm{ICH}(\mathrm{OR}=0.306)$ in the hospital and death event rates $(\mathrm{OR}=0.091)$ at 2 years. Using statins was not related to gastrointestinal haemorrhage in the hospital or FFO at 90 days. In severe stroke patients, statin use was significantly inversely related to ICH $(O R=0.278)$ in the hospital and death event rates $(\mathrm{OR}=0.204)$ at 2 years. Using statins was not related to NIHSS improvement or gastrointestinal haemorrhage in the hospital or FFO at 90 days (Fig. 06). In the cardioembolic stroke subgroup, statin use was significantly inversely related to death event rates $(O R=0.245)$ at 2 years. Using statins was not related to NIHSS improvement or ICH in the hospital or FFO at 90 days. In the noncardioembolic stroke subgroup, statin using was not related to NIHSS improvement $(\mathrm{OR}=1.938)$ or FFO at 90 days $(\mathrm{OR}=2.08)$ or gastrointestinal haemorrhage in the hospital. Statin using was significantly inversely related to $\mathrm{ICH}$ $(\mathrm{OR}=0.129)$ in the hospital and death event rates $(\mathrm{OR}=0.043)$ at 2 years (Fig. 07). 


\section{Discussion}

We found that the medium- and low-dose statin groups had a higher percentage of NIHSS improvement at 7 days after admission and a lower percentage of intracerebral haemorrhage and gastrointestinal haemorrhage in the hospital than the control group, and the medium-dose and low-dose groups did not show a significant difference. The two statin groups had a higher FFO and a lower percentage of death events within 2 years than the control group, and the medium- and low-dose statin groups did not show a significant difference. When we analysed risk factors through logistic regression, statins were significantly related to NIHSS improvement, fewer haemorrhage events in the hospital and death events within 2 years. When we evaluated death events through the K-M curve, we found that the two statin groups had fewer death events than the control group in both 2 years. The two statins did not have a significant difference in death rates before 20 months, after which the medium-dose group had fewer death events than the low-dose group. The effect of statins was more significant for moderate stroke patients or noncardioembolic stroke patients.

When the efficacy outcomes in the hospital were evaluated, our results were similar to those of Manuel Cappellari's study ${ }^{5}$, but the conclusion was not consistent with that of Ashkan Mowla's study ${ }^{6}$. Differences in study design could partly explain the difference. However, the effect was only significant for the moderate stroke subgroup or noncardioembolic stroke patients. Acute ischaemic patients after intravenous thrombolysis taking statins might have a short-term benefit for moderate stroke patients or noncardioembolic stroke patients. When evaluating functional outcomes at 90 days, our results showed that using statins was not significantly related to FFO. The difference in the NIHSS subgroup had a similar result. The results were consistent with those of the low-dose statin subgroup of Manuel Cappellari's study for 3 months of $\mathrm{FFO}^{5}$. The dose of statins might affect the longer-term outcome of ischaemic patients after intravenous thrombolysis. However, the noncardioembolic stroke subgroup showed a significant difference. Therefore, low- and medium-dose statins might improve FFO at 3 months in noncardioembolic stroke patients after intravenous thrombolysis. We found that a higher value of SBP at admission was a risk factor for higher mRS within 2 years, and the results were not entirely consistent with Anderson's study ${ }^{8}$. Anderson's intervention was intensive blood pressure reduction, and our data were SBP. The different data types could partly explain the different conclusions. The results suggest that blood pressure management at admission plays a role in the prognosis of ischaemic stroke patients with intravenous thrombolysis.

When we evaluated safety outcomes, we found that medium- and low-dose statins were related to fewer intracerebral haemorrhage and gastrointestinal haemorrhage events through the chi-square test and logistic regression. In the different subgroups, statin use was significantly inversely related to $\mathrm{ICH}$. It suggested the results had a statistic power. Our results showed that antiplatelet drug use, higher platelet counts and lower HDL-C levels were also related to fewer intracerebral haemorrhage events. The relationship between statin use and antiplatelet therapy was not consistent with that found in other studies $^{9,10}$. Patients not taking these drugs when they have an intracerebral haemorrhage, might partly explain the inconsistent results. It is easy to understand the effect of platelets on intracerebral 
haemorrhage but not that of HDL-C. The results for HDL-C were not consistent with other studies ${ }^{11}$. Previous studies evaluated symptomatic intracerebral haemorrhage, but we evaluated all intracerebral haemorrhages, which might partly explain the difference. We considered whether the higher value of HDL$\mathrm{C}$ had a similar mechanism to increase intracerebral haemorrhage as it decreased ischaemic stroke ${ }^{12}$. The results of reduced gastrointestinal haemorrhage were consistent with a study that included myocardial infarction patients ${ }^{13}$. This finding suggested that statins might be a protective factor against gastrointestinal haemorrhage in acute ischaemic stroke patients with intravenous thrombolysis. However, for the different subgroups, the relationship was not statistically significant. Therefore, further study is needed to confirm this conclusion. In addition, our results suggested that increasing age and higher blood glucose levels were related to more gastrointestinal haemorrhage. This suggests that we should consider these factors when evaluating the risk of intravenous thrombolysis for acute ischaemic stroke patients.

When evaluating long-term safety outcomes, we found that using statins resulted in fewer deaths over 2 years for acute ischaemic stroke patients with intravenous thrombolysis. The effect was significant for moderate and severe stroke patients. The effect was also significant for different stroke type patients. The results were similar to Manuel Cappellari's study about the effect of statins on the chance of death in acute ischaemic stroke patients with intravenous thrombolysis over 3 months ${ }^{6}$. Our results suggested that using statins to decrease death events had a longer-term effect. Within 2 years, the medium- and lowdose statin groups both had significantly fewer deaths than the control group. We also found that the medium-dose group had a lower mortality rate than the low-dose group after 20 months. Although the difference was not statistically significant, it suggested that the dose of statins might alter the effect of decreasing long-term mortality for acute ischaemic stroke patients with intravenous thrombolysis. Nonetheless, more research is needed to confirm this conclusion.

Our study has several limitations. First, the study could not identify a causal relationship given the observational cohort design. However, the conditions of the prospective observational cohort were consistent with those of the real world, and the conclusions might be suitable for use in clinical practice. Second, the long-term outcome and event data might be affected by risk factors that we did not collect or by memory bias. Although we collected follow-up data through different pathways, these biases could not be entirely avoided. The smaller number of patients in the control groups might have affected the statistical power of the results. Although we used logistic regression to increase the statistical power of the results, a study including more control-group patients could further support the conclusions of the present study.

In conclusion, for Asian acute ischaemic patients after intravenous thrombolysis, the use of statins was related to NIHSS score improvement at 7 days. The use of statins was related to improved FFO at 90 days only for the noncardioembolic stroke subgroup. The use of statins was related to a lower percentage of intracerebral haemorrhage and gastrointestinal haemorrhage in the hospital and to lower death event rates within 2 years. These relationships were significant, especially for moderate stroke patients and noncardioembolic stroke patients. The use of medium-dose statins might result in lower long-term mortality than low-dose statins, but this conclusion needs further study to be proven. 


\section{Declarations}

\section{Ethics approval and consent to participate}

The study was performed in accordance with the Declaration of Helsinki and the ethical standards of the institutional and national research committees. The study was approved by the Ethics Committee of West China Hospital, Sichuan University, under approval number 2019 (319). Informed consent was obtained from all individual participants included in the study.

\section{Consent for publication:}

The manuscript has been approved by all authors for publication.

\section{Availability of data and materials $\rrbracket$}

The datasets used or analysed during the current study are available from the corresponding author on reasonable request.

\section{Competing interests $\llbracket$}

All authors declare no conflicts of interest or competing interests.

\section{Funding}

This study was supported by the project "National Key R\&D Program of China" (Funding number: 2018YFC1311400 and 2018YFC1311401) and the project "National Natural Science Foundation of China" (Funding number: 81772435).

\section{Authors' contributions}

Professor he and Dr cui conceived and designed the study. Dr cui, MD Bao, and Dr Gao screened and collected data for the article. Dr cui and MD Bao conducted statistical analyses and written the manuscript. All authors reviewed and revised the paper.

\section{Acknowledgements:}

We thank Professor Ning Chen for offering some guidance for the design of the study. 


\section{References}

1. Powers. 2018 Guidelines for the Early Management of Patients With Acute Ischemic Stroke: A Guideline for Healthcare Professionals From the American Heart Association/American Stroke Association (vol 49, pg e46, 2018). Stroke 2018; 49: E138-E138. DOI:

$10.1161 /$ str.0000000000000163.

2. Makihara N, Okada Y, Koga M, et al. Effect of Serum Lipid Levels on Stroke Outcome after rt-PA Therapy: SAMURAI rt-PA Registry. Cerebrovascular Diseases 2012; 33: 240-247. DOI: $10.1159 / 000334664$.

3. Wahlgren N, Ahmed N, Davalos A, et al. Thrombolysis with alteplase for acute ischaemic stroke in the Safe implementation of Thrombolysis in Stroke-Monitoring Study (SITS-MOST): an observational study. Lancet 2007; 369: 275-282. DOI: 10.1016/s0140-6736(07)60149-4.

4. Amarenco P, Bogousslavsky J, Callahan A, et al. High-dose atorvastatin after stroke or transient ischemic attack. New England Journal of Medicine 2006; 355: 549-559.

5. Wu YS, Lu D and Xu AD. The effect of HMG-CoA reductase inhibitors on thrombolysis-induced haemorrhagic transformation. Journal of Clinical Neuroscience 2019; 69: 1-6. DOI: 10.1016/j.jocn.2019.08.074.

6. Mowla A, Shah H, Lail NS, et al. Statins Use and Outcome of Acute Ischemic Stroke Patients after Systemic Thrombolysis. Cerebrovasc Dis 2020: 1-6. 2020/09/15. DOI: 10.1159/000510095.

7. Cappellari M, Bovi P, Moretto G, et al. The THRombolysis and STatins (THRaST) study. Neurology 2013; 80: 655-661. DOI: 10.1212/WNL.0b013e318281cc83.

8. Anderson CS, Huang YN, Lindley RI, et al. Intensive blood pressure reduction with intravenous thrombolysis therapy for acute ischaemic stroke (ENCHANTED): an international, randomised, openlabel, blinded-endpoint, phase 3 trial. Lancet 2019; 393: 877-888. DOI: 10.1016/s01406736(19)30038-8.

9. Lin T-C, Lin Y-K, Chen C-I, et al. Serum lipid level is not associated with symptomatic intracerebral hemorrhage after intravenous thrombolysis for acute ischemic stroke. Peerj 2018; 6. DOI: 10.7717/peerj.6021.

10. Rocco A, Sykora M, Ringleb P, et al. Impact of Statin Use and Lipid Profile on Symptomatic Intracerebral Haemorrhage, Outcome and Mortality after Intravenous Thrombolysis in Acute Stroke. Cerebrovascular Diseases 2012; 33: 362-368. DOI: 10.1159/000335840.

11. Nardi K, Engelter S, Strbian D, et al. Lipid profiles and outcome in patients treated by intravenous thrombolysis for cerebral ischemia. Neurology 2012; 79: 1101-1108. DOI:

10.1212/WNL.0b013e3182608c82.

12. Zhang Y, Tuomilehto J, Jousilahti P, et al. Total and High-Density Lipoprotein Cholesterol and Stroke Risk. Stroke 2012; 43: 1768-1774. DOI: 10.1161/strokeaha.111.646778.

13. Atar S, Cannon CP, Murphy SA, et al. Statins are associated with lower risk of gastrointestinal bleeding in patients with unstable coronary syndromes: Analysis of the orbofiban in patients with 
unstable coronary syndromes-thrombolysis in myocardial infarction 16 (OPUS-TIMI 16) trial. American Heart Journal 2006; 151. DOI: 10.1016/j.ahj.2006.02.013.

14. Tramacere I, Boncoraglio GB, Banzi R, et al. Comparison of statins for secondary prevention in patients with ischemic stroke or transient ischemic attack: a systematic review and network metaanalysis. Bmc Medicine 2019; 17. DOI: 10.1186/s12916-019-1298-5.

Figures

\section{Efficacy Outcome by Univariate Logistic Regression}

OR $(95 \% \mathrm{Cl})$

\section{NIHSS Improvement}

using statins

uising antiplatelet

$2.89(1.69,4.94)$

\section{FFO}

using statins

uising antiplatelet

older

higher SBP

higher NIHSS at admission

higher NIHSS at 7 days

$\mathrm{ICH}$ in hospital

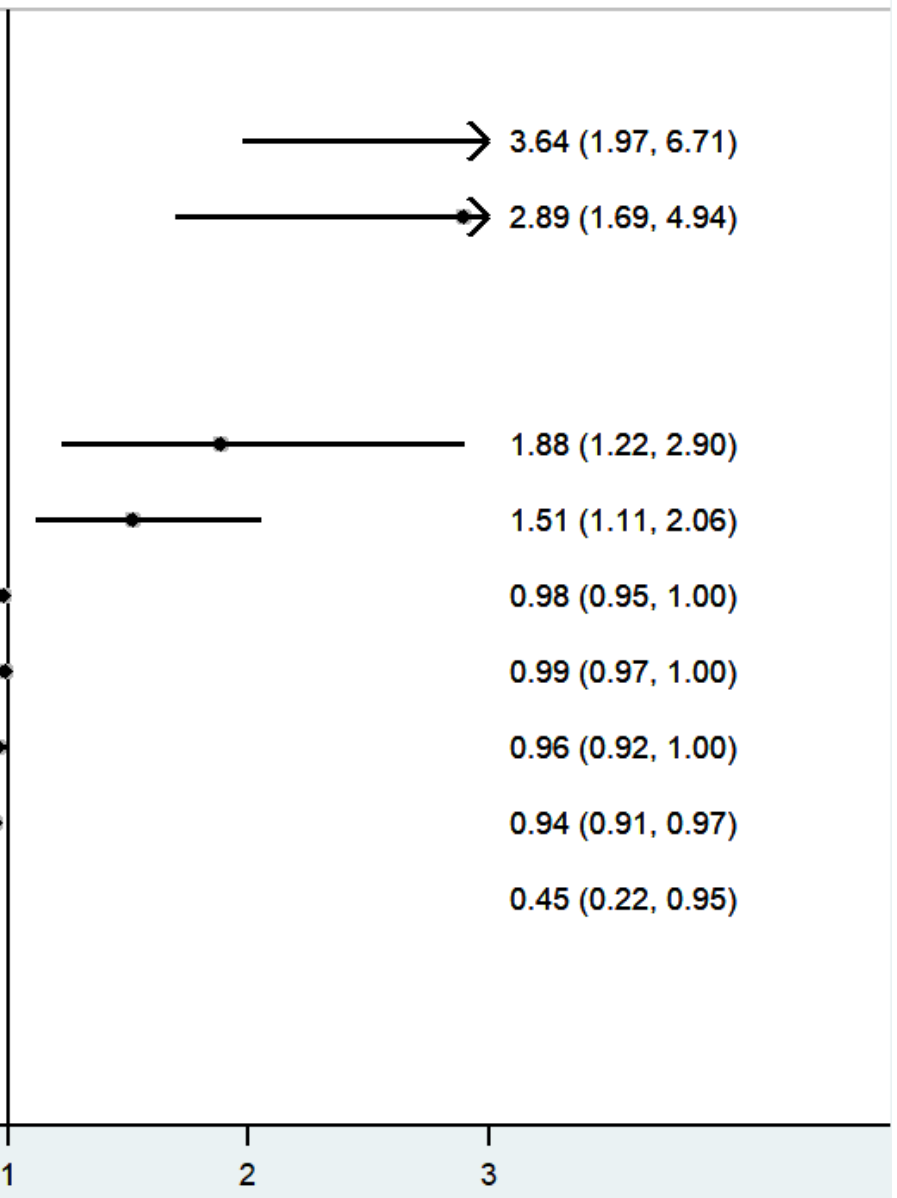

\section{Figure 1}

Efficacy outcome by univariate logistic regression analysis 


\section{Efficacy Outcome by Multivariable Logistic Regression}

OR $(95 \% \mathrm{Cl})$

\section{NIHSS Improvement}

using statins

uising antiplatelet

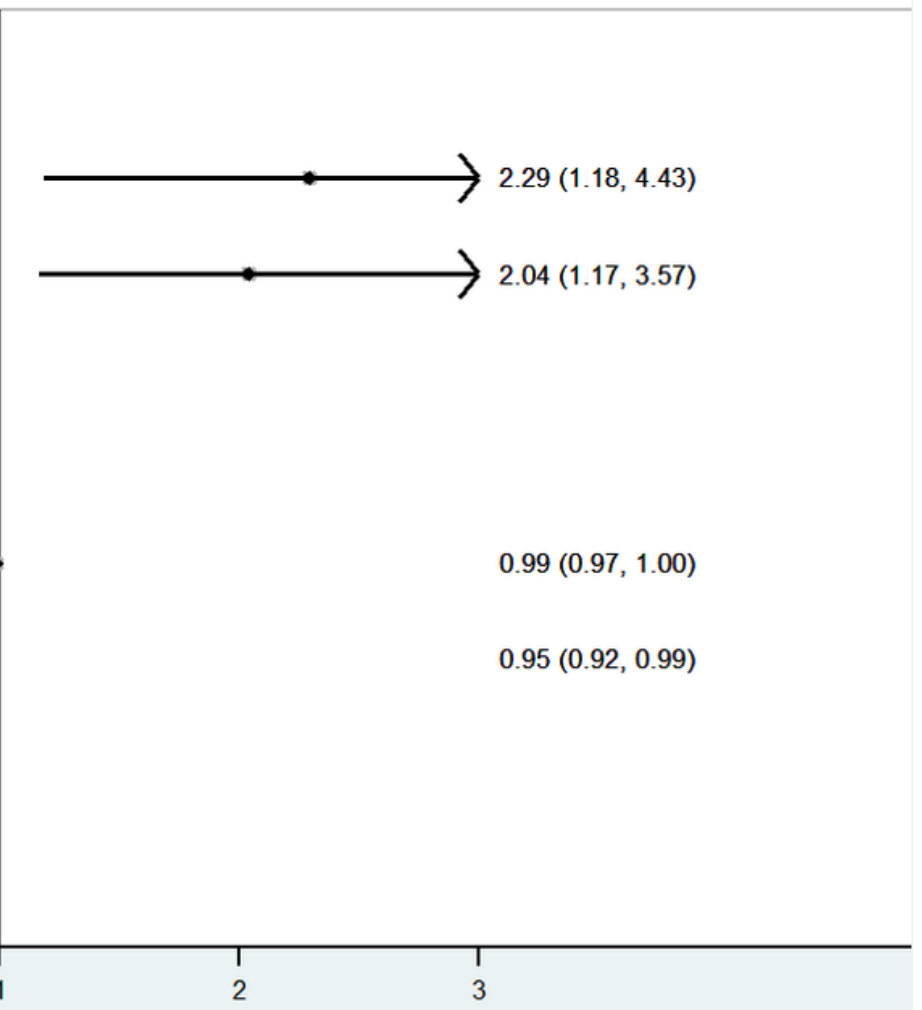

FFO

higher SBP

higher NIHSS at 7 days

Figure 2

Efficacy outcome by multivariable logistic regression analysis 


\section{Safety Outcome by Univariate Logistic Regression}

OR $(95 \% \mathrm{Cl})$

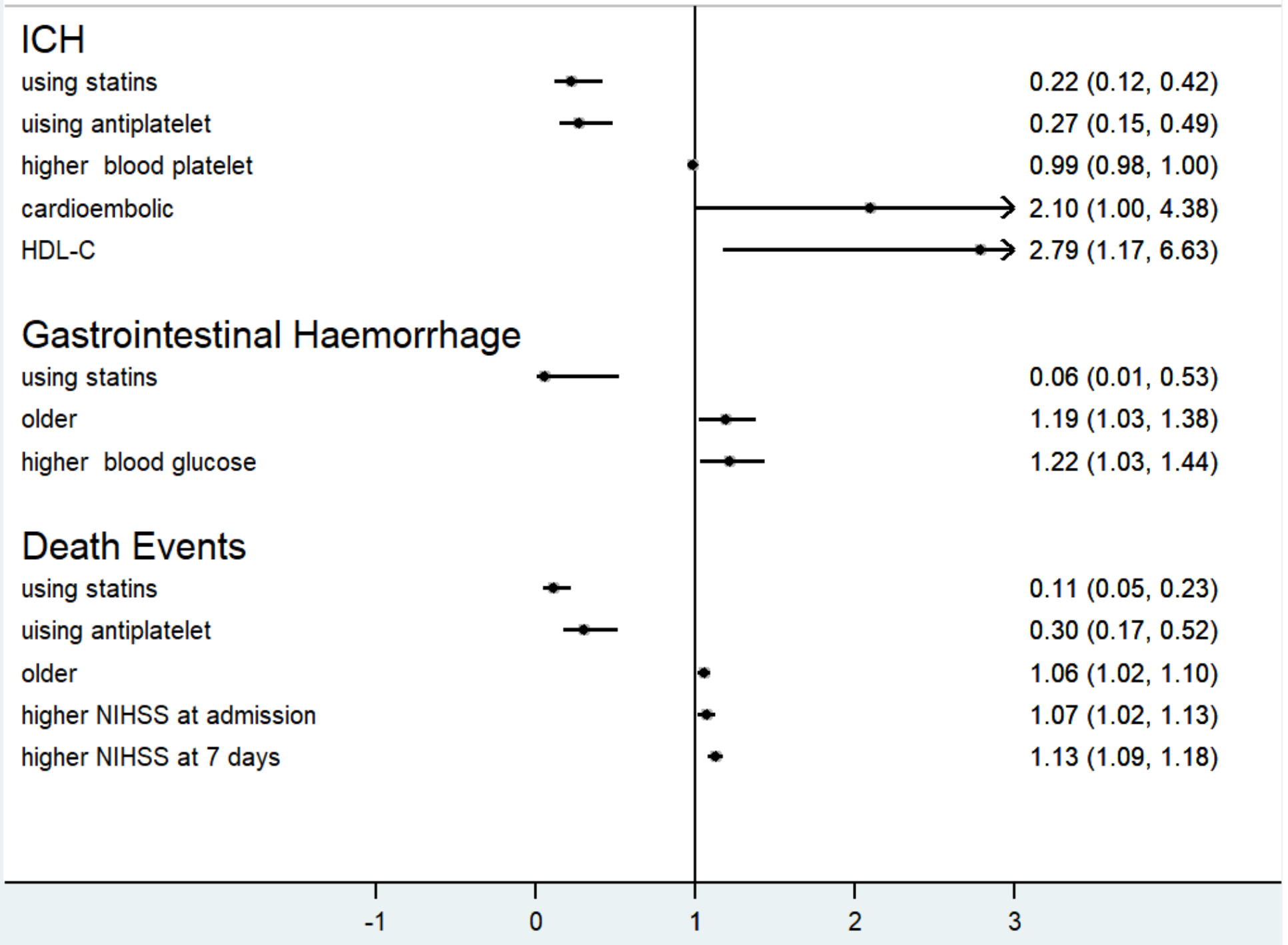

Figure 3

Safety outcome by univariate logistic regression analysis 


\section{Safety Outcome by Multivariable Logistic Regression}

OR $(95 \% \mathrm{Cl})$

\section{$\mathrm{ICH}$}

using statins

uising antiplatelet

higher blood platelet

HDL-C

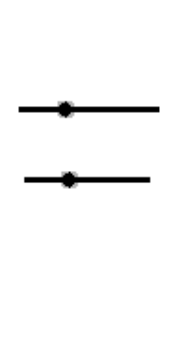

$0.38(0.19,0.77)$

$0.40(0.21,0.74)$

$0.99(0.98,1.00)$

$3.13(1.09,8.97)$

Gastrointestinal Haemorrhage

using statins

older

higher blood glucose

\section{Death Events}

using statins

older

higher NIHSS at 7 days

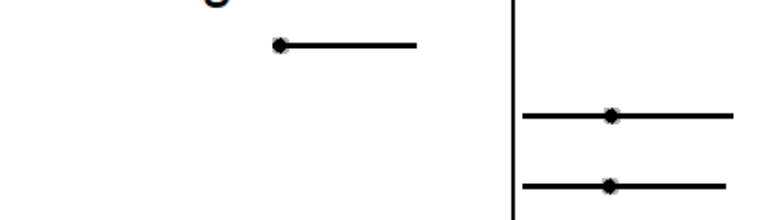

$0.03(0.00,0.60)$

$1.41(1.04,1.92)$

$1.40(1.03,1.89)$

$0.20(0.08,0.47)$

$1.05(1.01,1.10)$

$1.09(1.04,1.15)$

Figure 4

Safety outcome by multivariable logistic regression analysis 


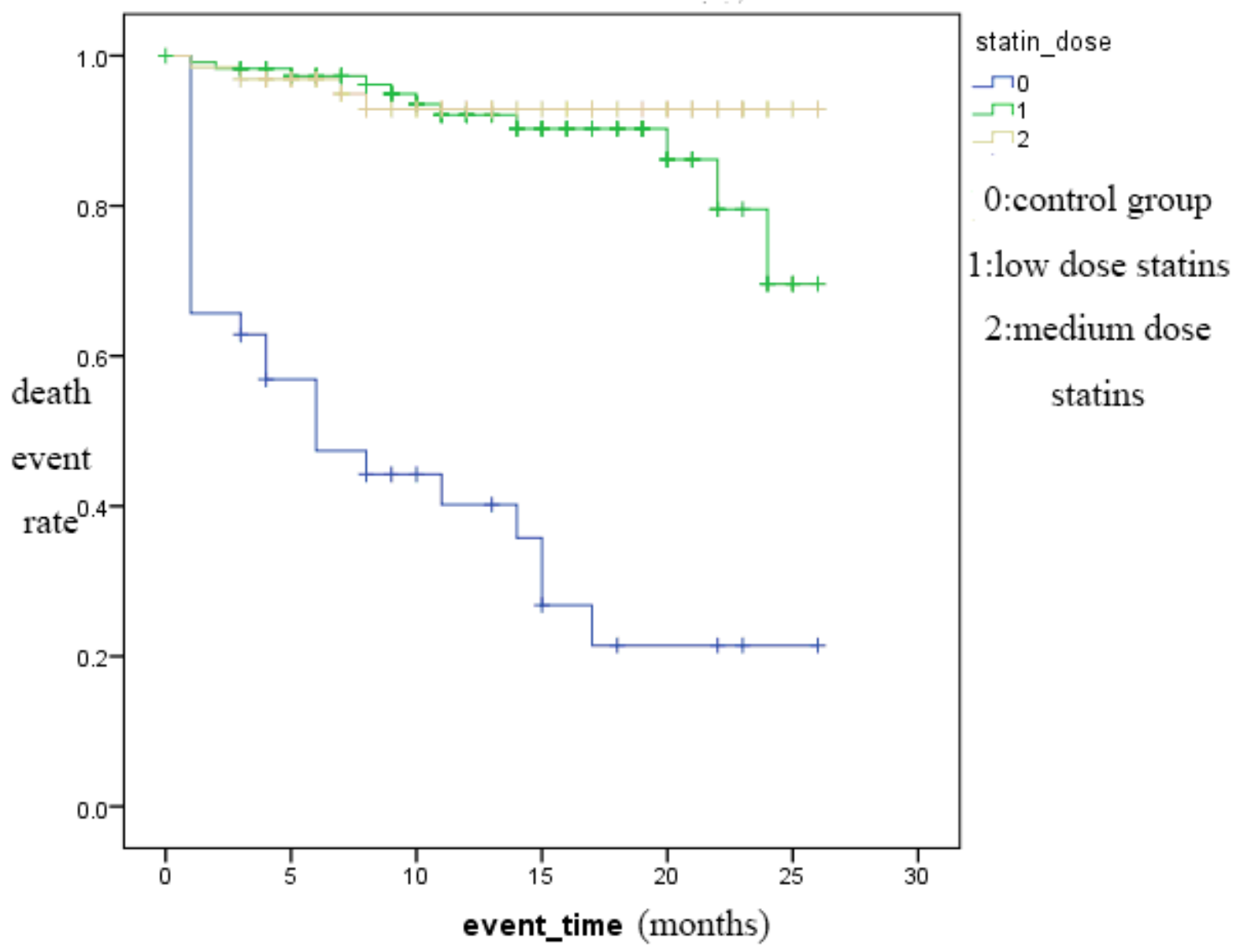

Figure 5

Death events by Kaplan-Meier curve 


\section{Subgroup Analysis By Difference NIHSS At Admission}

OR $(95 \% \mathrm{Cl})$

\section{mild stroke(NIHSS 0-4)}

NIHSS_Improvement

FFO

$\mathrm{ICH}$

gastrointestinal haemorrhage

Higher Death Rates

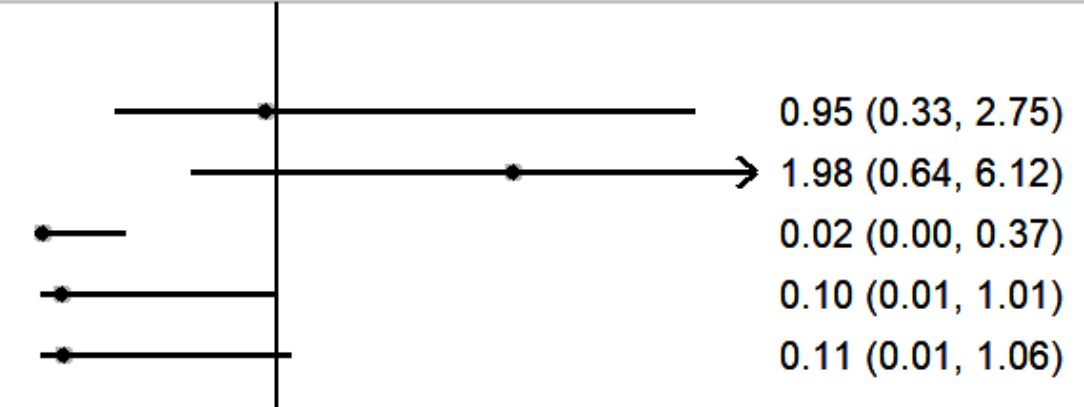

moderate stroke(NIHSS 5-15)

NIHSS_Improvement

FFO

$\mathrm{ICH}$

gastrointestinal haemorrhage

Higher Death Rates

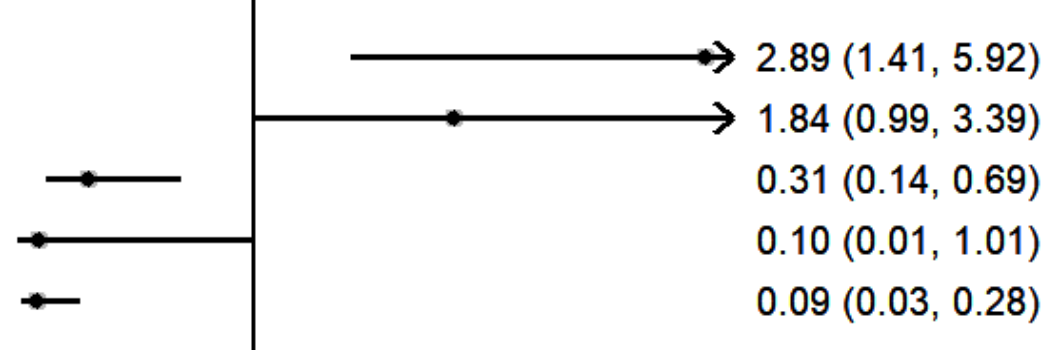

severe stroke(NIHSS $\geq 16$ )

NIHSS_Improvement

FFO

$\mathrm{ICH}$

Higher Death Rates

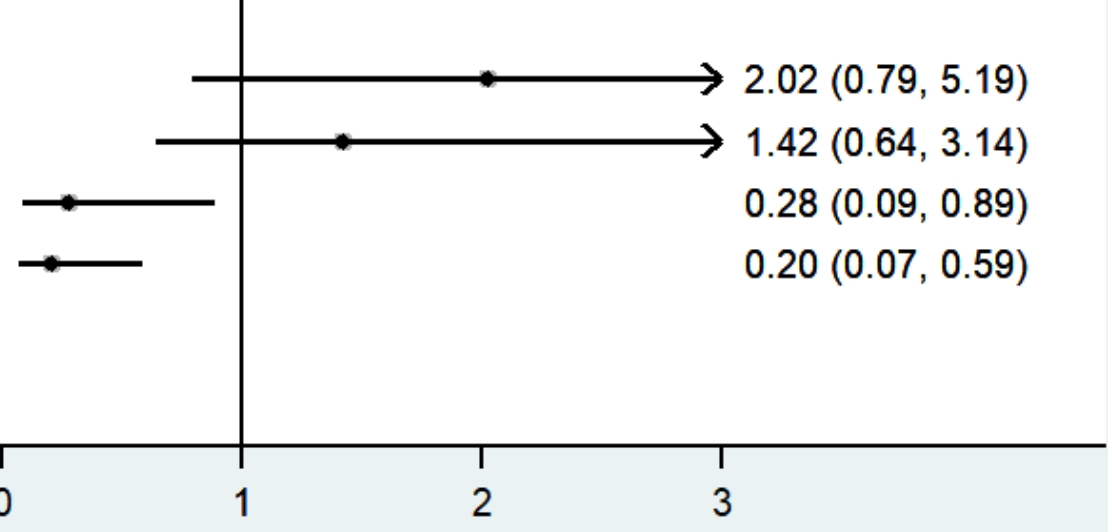

Figure 6

Subgroup analysis by differences in NIHSS scores 


\section{Subgroup Analysis By Difference Stroke Type}

\section{$\mathrm{OR}(95 \% \mathrm{Cl})$}

\section{Non-Cardioembolic}

NIHSS_Improvement

FFO

$\mathrm{ICH}$

gastrointestinal haemorrhage

Higher Death Rates

\section{Cardioembolic}

NIHSS_Improvement

FFO

$\mathrm{ICH}$

Higher Death Rates
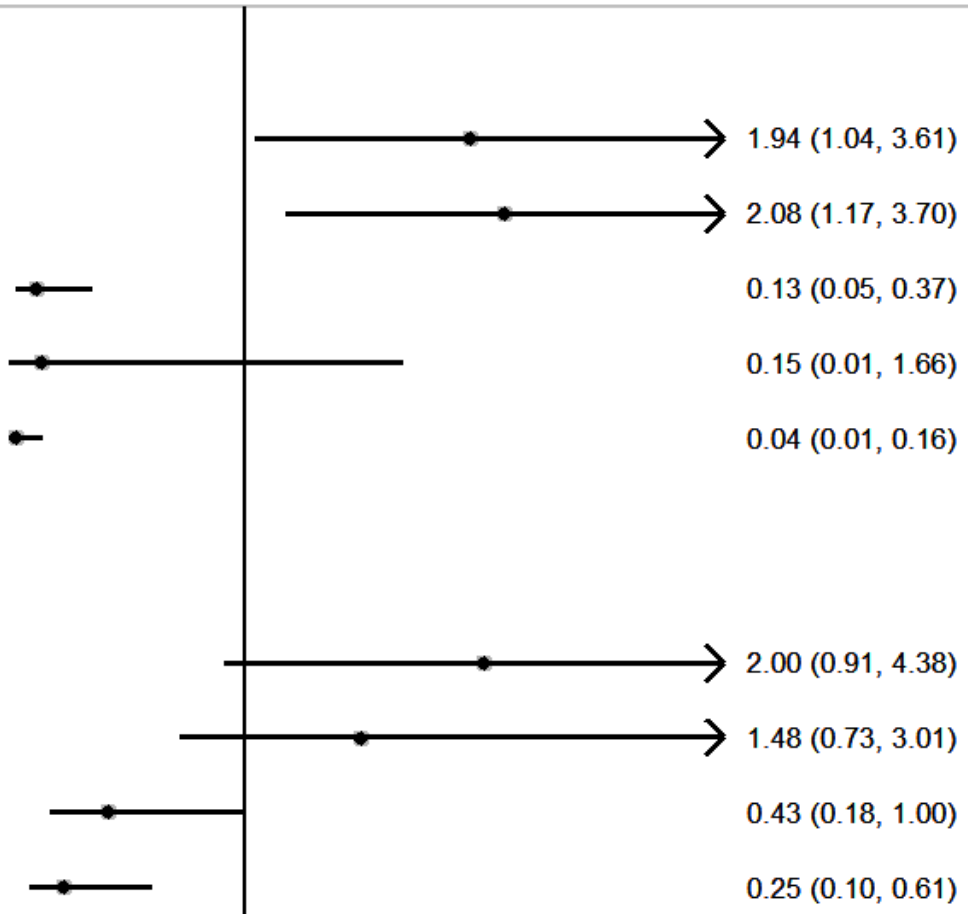

\section{$-1$}

0

1

3

Figure 7

Subgroup analysis by different stroke types 